Supplement of Biogeosciences, 14, 827-859, 2017

http://www.biogeosciences.net/14/827/2017/

doi:10.5194/bg-14-827-2017-supplement

(C) Author(s) 2017. CC Attribution 3.0 License.

(c) (i)

Supplement of

\title{
Factors controlling the depth habitat of planktonic foraminifera in the subtropical eastern North Atlantic
}

\author{
Andreia Rebotim et al. \\ Correspondence to: Andreia Rebotim (andreia.rebotim@ipma.pt)
}

The copyright of individual parts of the supplement might differ from the CC-BY 3.0 licence. 


\section{POS 377}

\section{Station 696}

N. incompta G. ruber (white) T. sacculifer G. truncatulinoides G. glutinata G. siphonifera G. calida T. quinqueloba G. falconensis G. rubescens

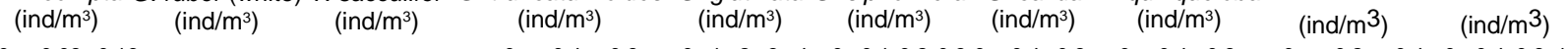
$\begin{array}{llllll}0 & 0.08 & 0.16 & 0 & 0.8 & 1.6\end{array}$
$\begin{array}{lll}0 & 0.1 & 0.2\end{array}$
$0 \quad 0.4 \quad 0.8$
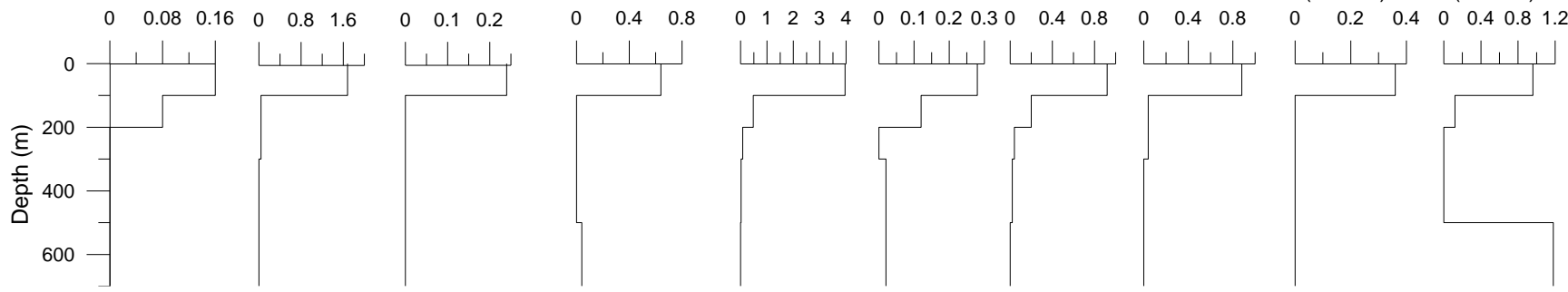

\section{Station 704}

N. incompta G. ruber (white) G. truncatulinoides G. glutinata G. siphonifera G. calida T. quinqueloba G. bulloides T. parkerae G. falconensis

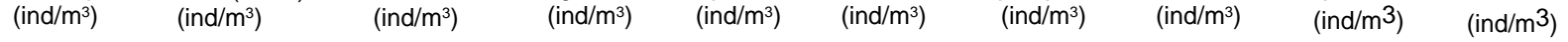
$\begin{array}{llllll}0 & 0.2 & 0.4 & 0 & 0.8 & 1.6\end{array}$
$\begin{array}{llll}0 & 1 & 2 & 3\end{array}$
$\begin{array}{lll}0 & 0.2 & 0.4\end{array}$
$\begin{array}{lllll}0.08 & 0.16 & 0 & 0.08 & 0.16\end{array}$
0.10 .2

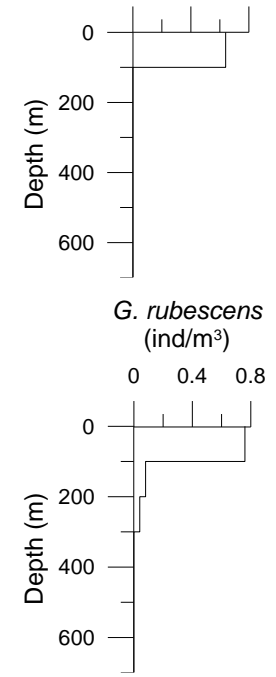




\section{POS 383}

\section{Station 161}

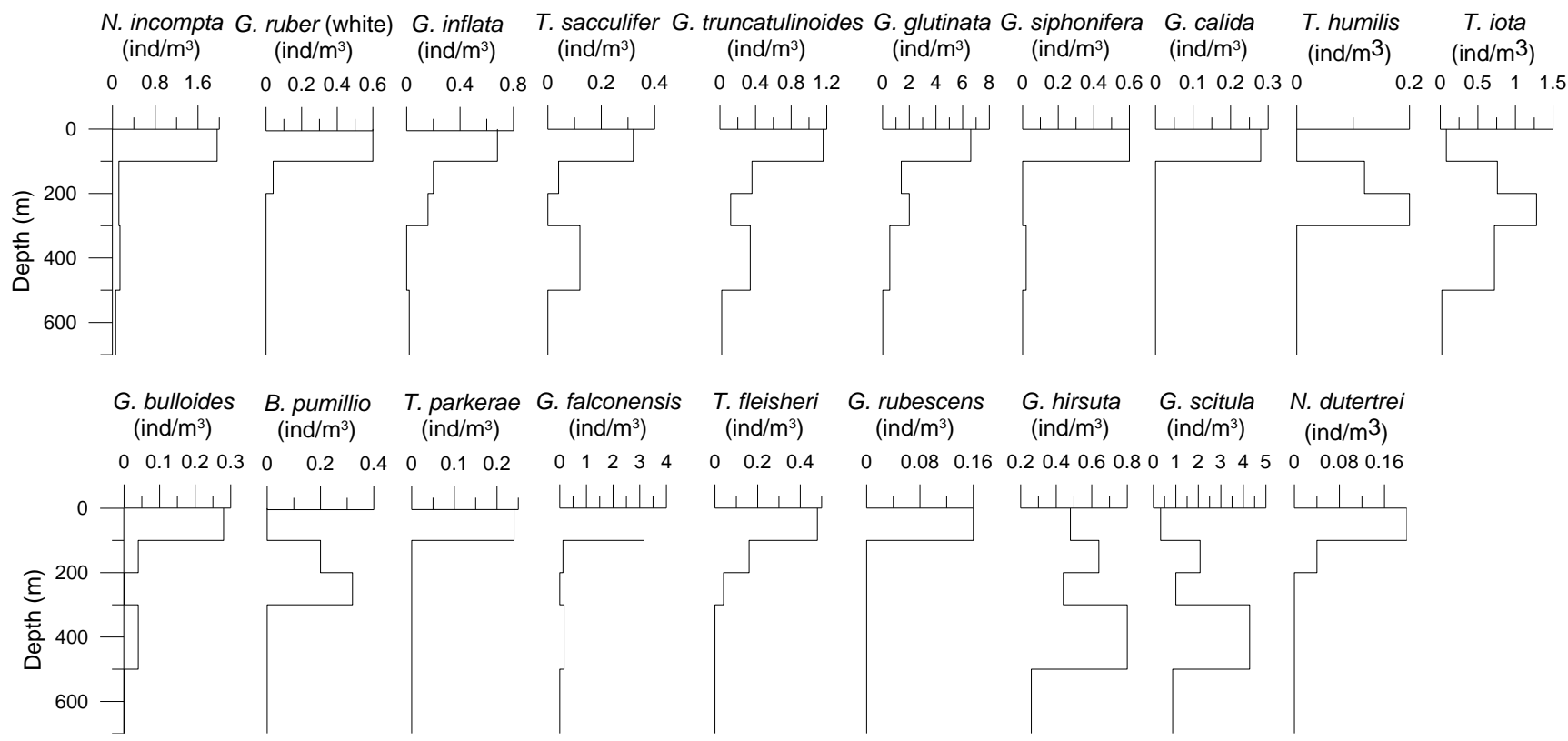

\section{Station 163}

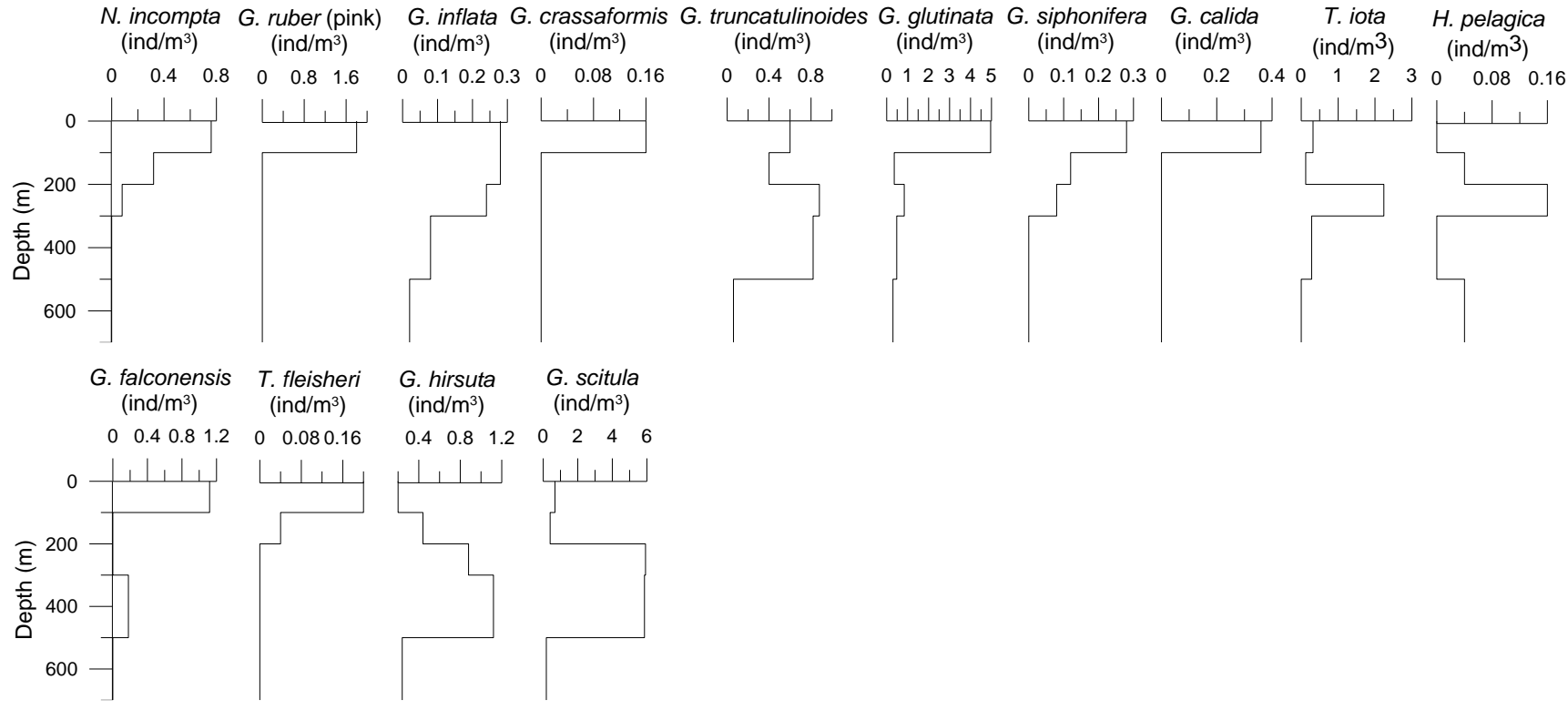




\section{POS 383}

\section{Station 165}

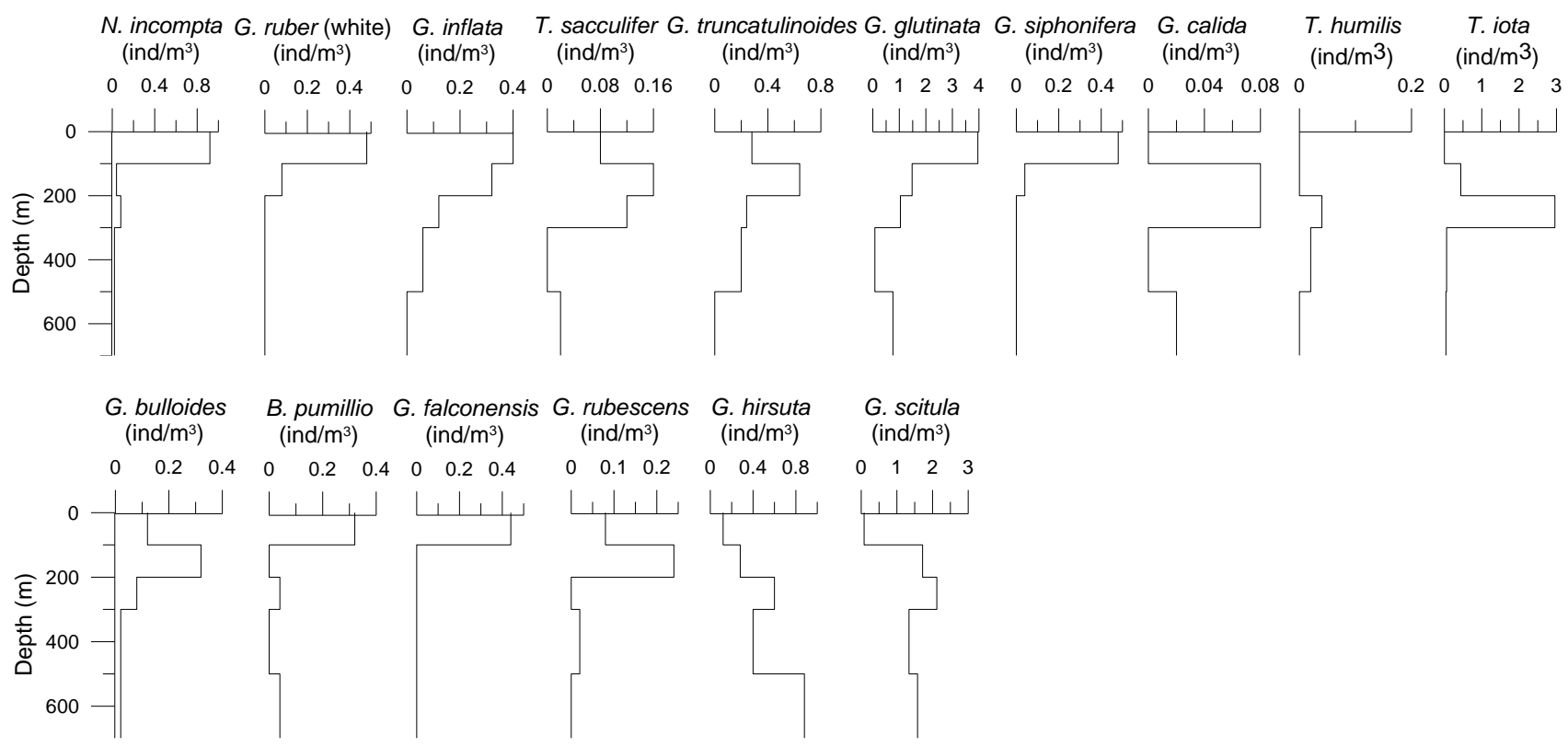

\section{Station 173}

N. incompta G. ruber (white) G. ruber (pink) G. inflata T. sacculifer G. truncatulinoides G. glutinata G. siphonifera G. calida T. iota $\quad\left(\right.$ ind $\left./ \mathrm{m}^{3}\right) \quad\left(\right.$ ind $\left./ \mathrm{m}^{3}\right)$

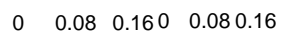
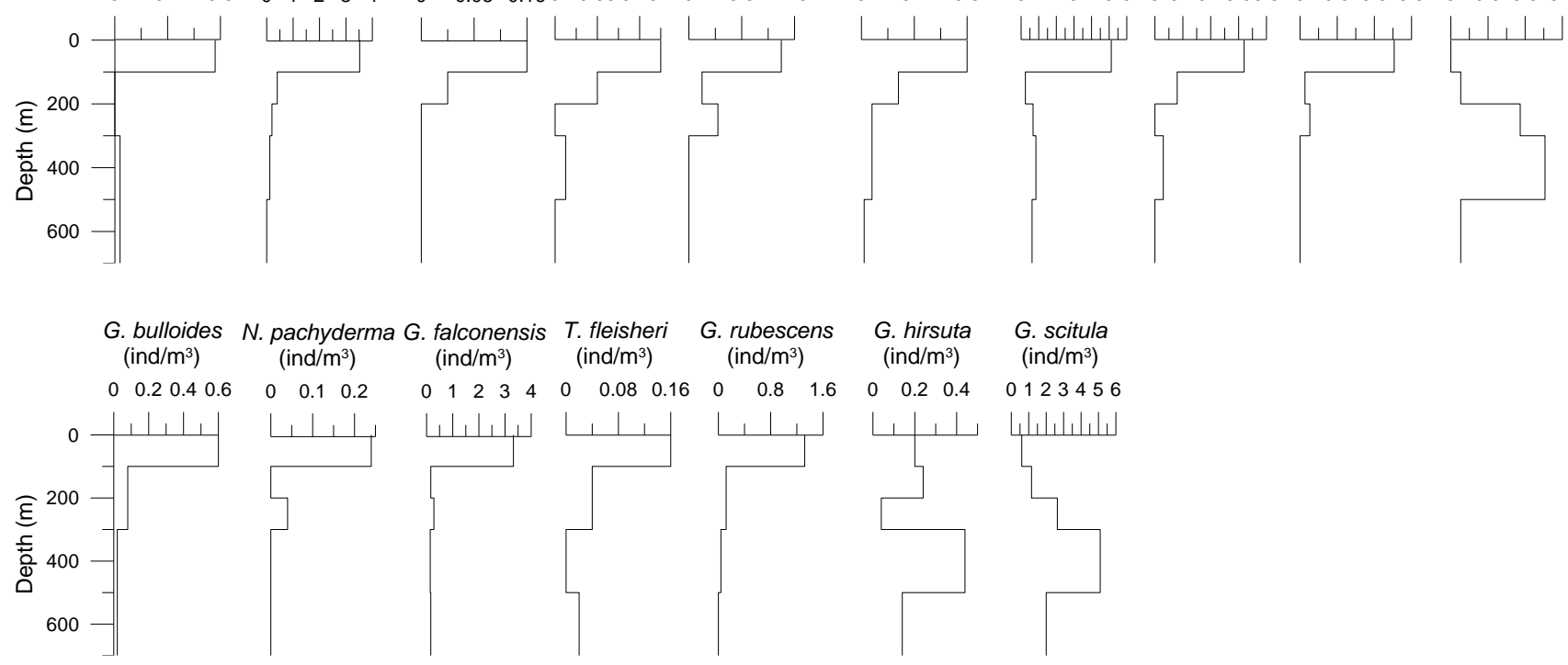
POS 383

\section{Station 175}

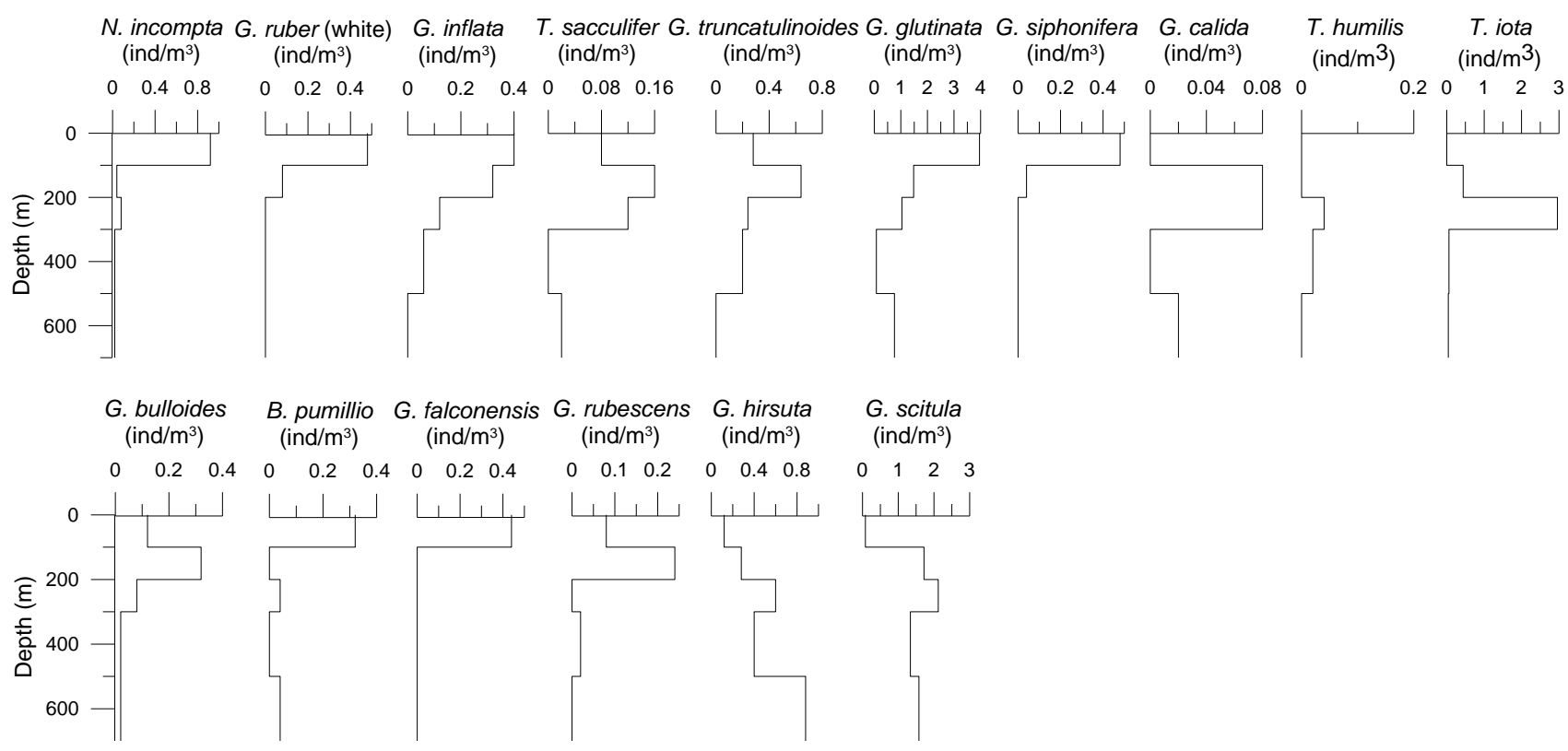


POS 384

\section{Station 210}

G. ruber (white)

G. inflata

T. sacculifer

G. glutinata (ind $/ \mathrm{m}^{3}$ ) (ind $/ \mathrm{m}^{3}$ ) (ind $/ \mathrm{m}^{3}$ ) (ind $/ \mathrm{m}^{3}$ )

G. siphonifera $N$. pachyderma 0 . universa (ind $/ \mathrm{m}^{3}$ ) (ind $/ \mathrm{m}^{3}$ ) (ind $/ \mathrm{m}^{3}$ )

G. rubescens

$\begin{array}{lll}0 & 0.4 & 0.8\end{array}$

$\begin{array}{lll}0 & 0.1 & 0.2\end{array}$

$\begin{array}{lll}0 & 0.1 & 0.2\end{array}$

$\begin{array}{lll}0 & 0.4 & 0.8\end{array}$

$\begin{array}{lll}0 & 0.1 & 0.2\end{array}$

$\begin{array}{llllll}0 & 0.06 & 0.12 & 0 & 0.04 & 0.08\end{array}$

(ind $/ \mathrm{m}^{3}$ )
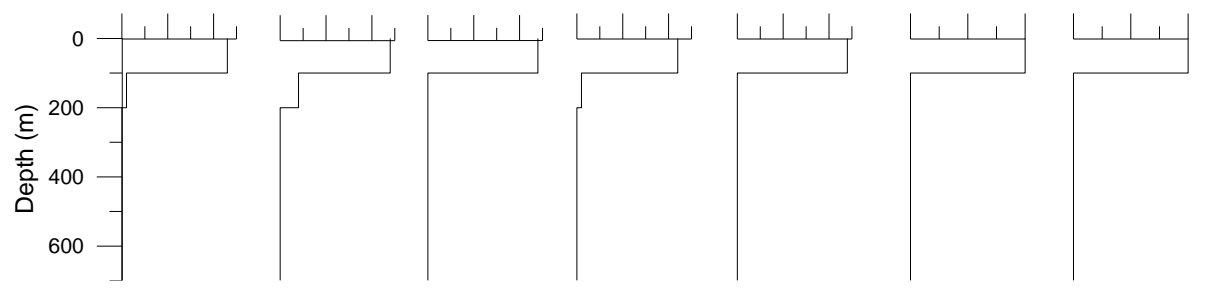

\section{Station 273}

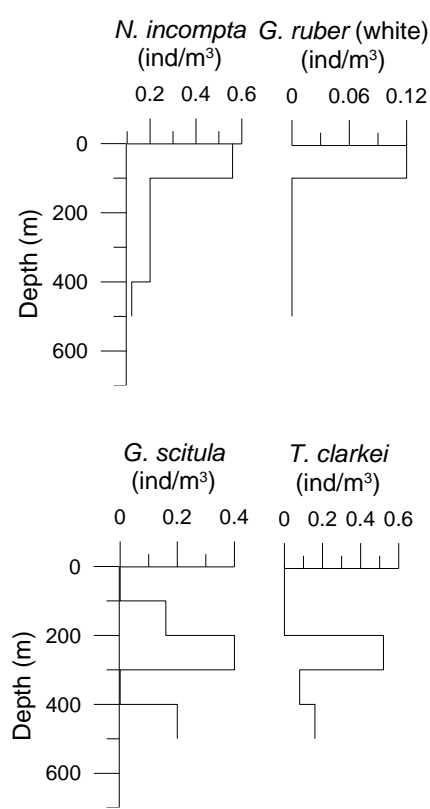
G. inflata T. sacculifer G. glutinata T. quinqueloba T. iota

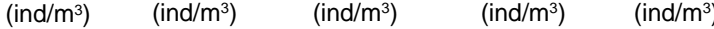
B. pumillio T.parkerae G. falconensis (ind $\left./ \mathrm{m}^{3}\right) \quad\left(\right.$ ind $\left./ \mathrm{m}^{3}\right)$
(ind $/ \mathrm{m}^{3}$ )
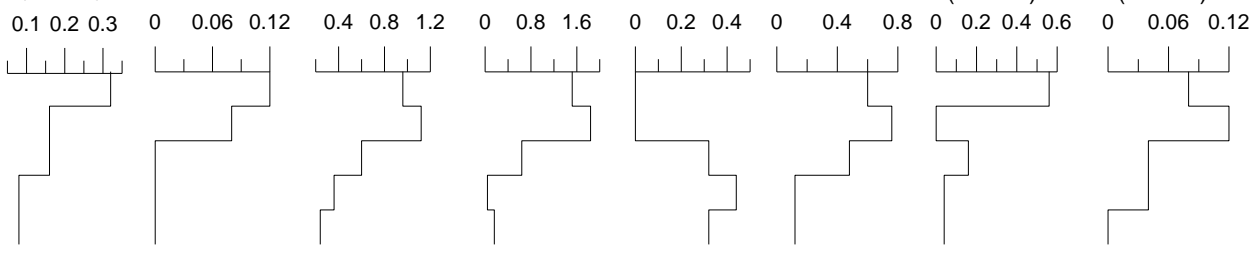


\section{Iberia-Forams}

\section{Station Ib-F2}

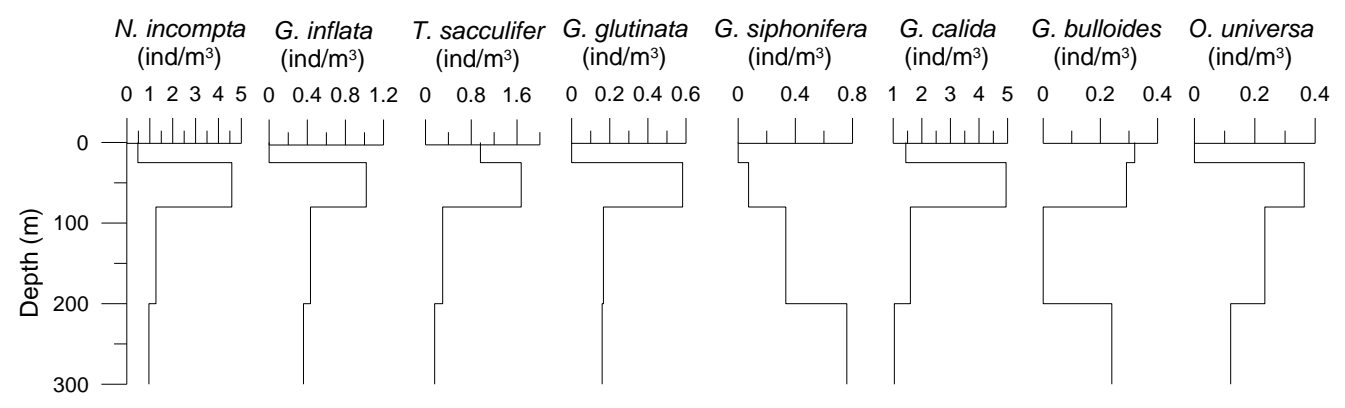

\section{Station Ib-F6}

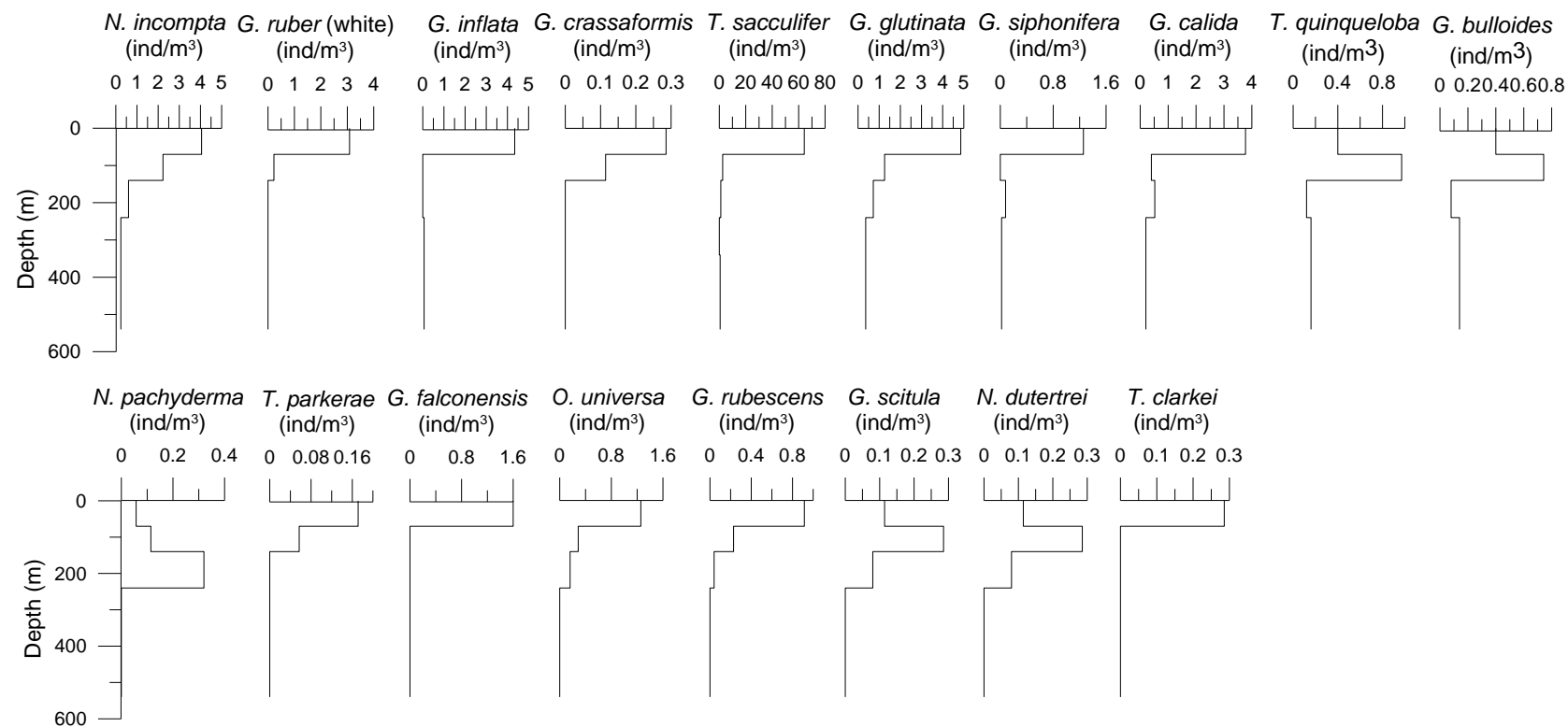

\section{Station Ib-F8}
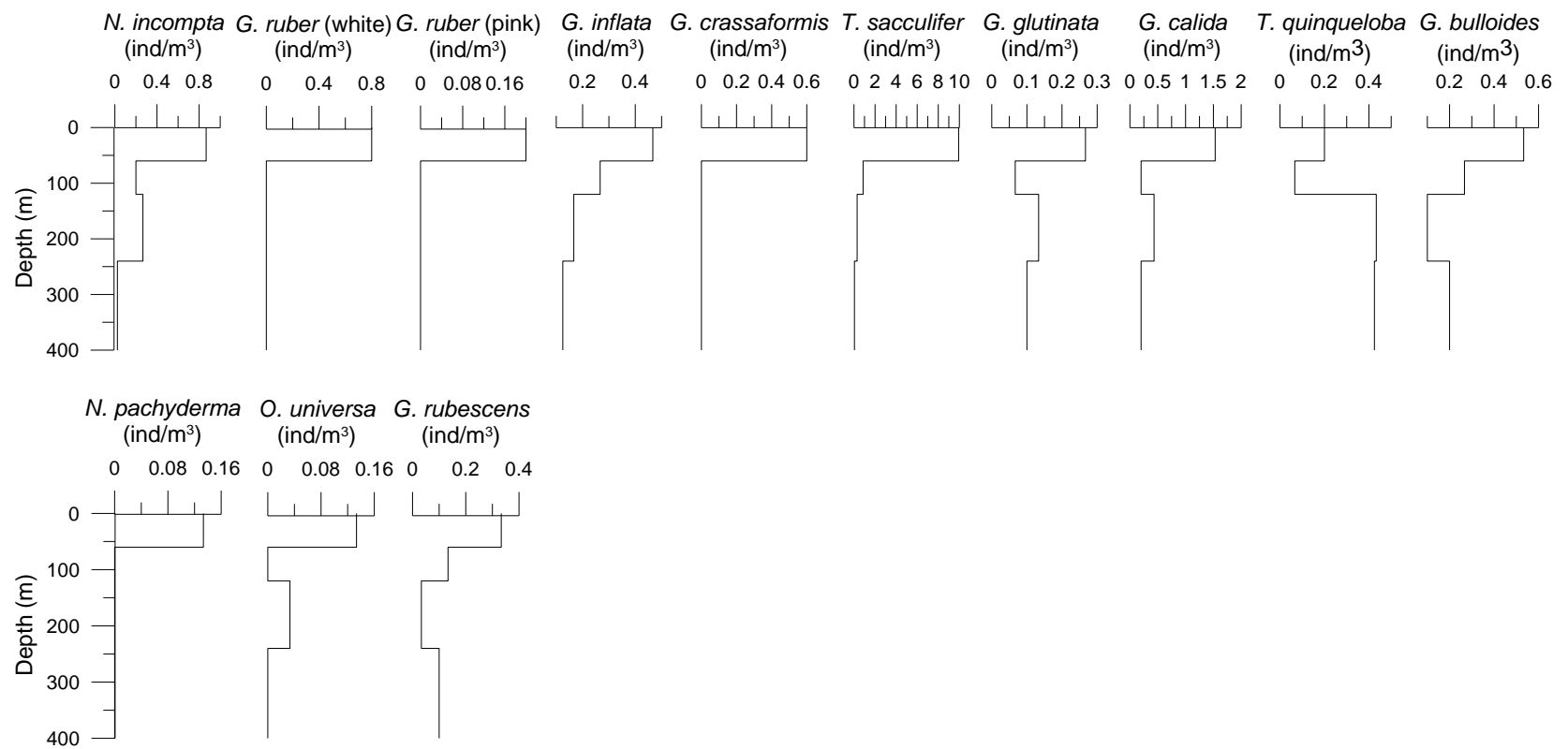


\section{Iberia-Forams}

\section{Station Ib-F9}

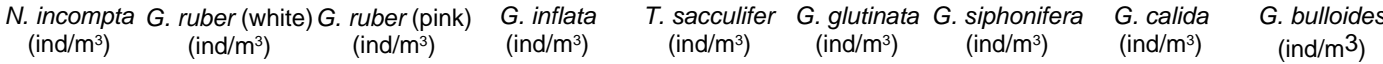

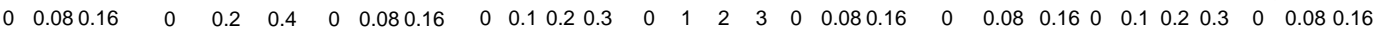

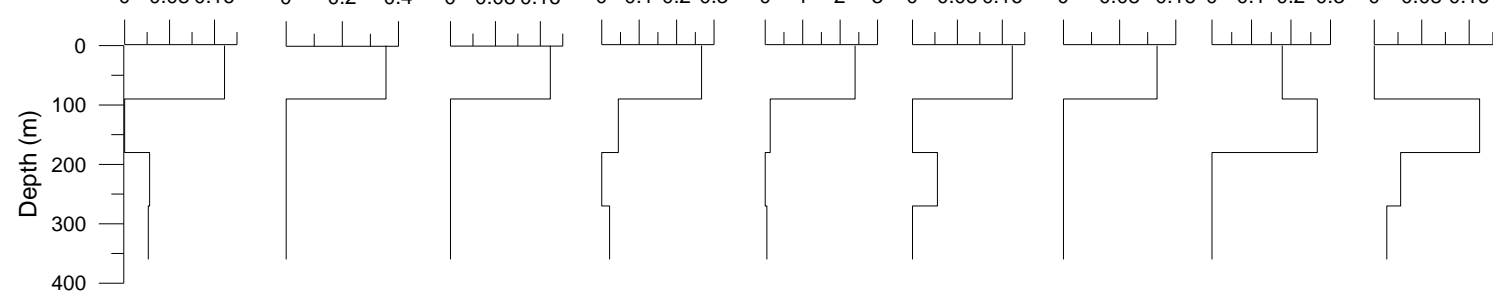

\section{Station Ib-F12} $\begin{array}{llllllllll}\left(\text { ind } / \mathrm{m}^{3}\right) & \left(\text { ind } / \mathrm{m}^{3}\right) & \left(\mathrm{ind} / \mathrm{m}^{3}\right) & \left(\mathrm{ind} / \mathrm{m}^{3}\right) & \left(\mathrm{ind} / \mathrm{m}^{3}\right) & \left(\mathrm{ind} / \mathrm{m}^{3}\right) & \left(\mathrm{ind} / \mathrm{m}^{3}\right) & \left(\mathrm{ind} / \mathrm{m}^{3}\right) & \left(\mathrm{ind} / \mathrm{m}^{3}\right)\end{array}$
T. quinqueloba

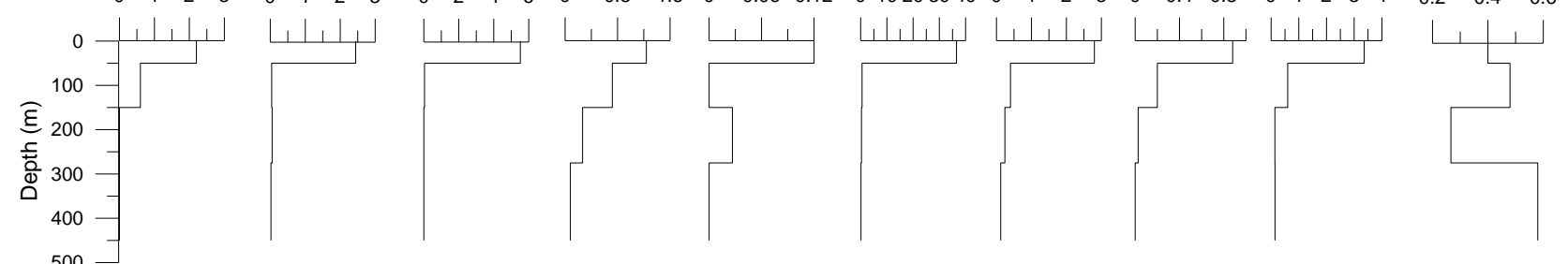

G. bulloides $G$. falconensis O. universa $G$. rubescens $\begin{array}{lllll}\left.\text { (ind } / \mathrm{m}^{3}\right) & \left.\text { (ind } / \mathrm{m}^{3}\right) & \left(\text { ind } / \mathrm{m}^{3}\right) & \left.\text { (ind } / \mathrm{m}^{3}\right)\end{array}$

$\begin{array}{llllllllllllll}0 & 4 & 8 & 12 & 0 & 0.4 & 0.8 & 1.2 & 0 & 0.4 & 0.8 & 0 & 0.2 & 0.4\end{array}$

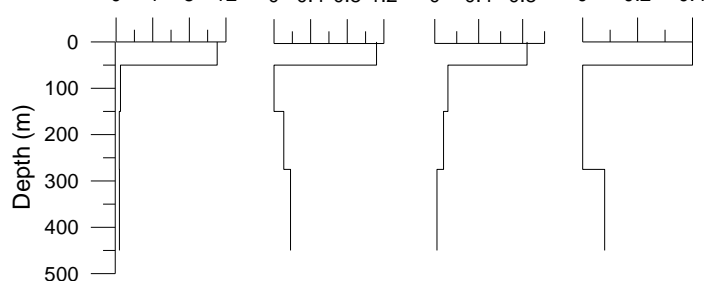


POS 334

\section{Station 67}

N. incompta G. ruber (white) G. inflata T. sacculifer G. truncatulinoides G. glutinata G. siphonifera G. calida T. humilis T. quinqueloba

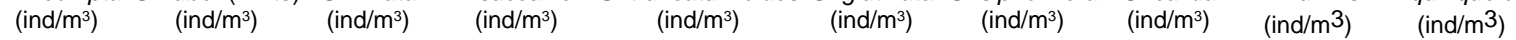

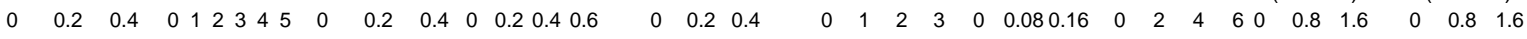

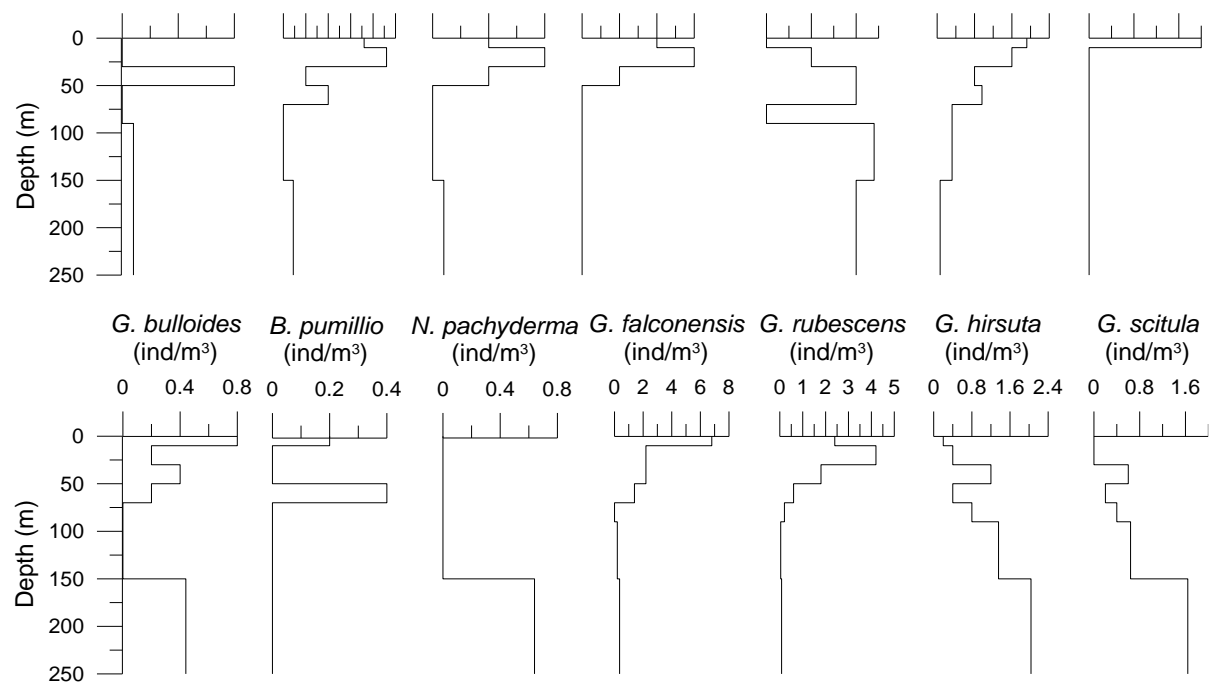

\section{Station 72}

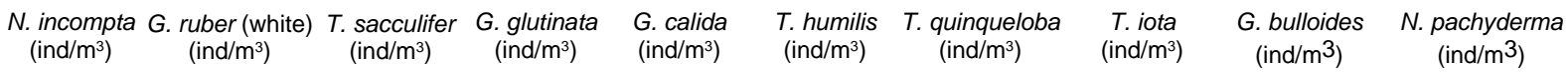

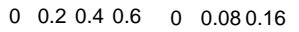
$\begin{array}{lllllllllll}0 & 0.2 & 0.4 & 0 & 1 & 2 & 3 & 4 & 0 & 0.4 & 0.8\end{array}$
$\begin{array}{lll}0 & 1 & 2\end{array}$
$\begin{array}{lll}0 & 0.1 & 0.2\end{array}$

$\begin{array}{llllllllll}0 & 1 & 3 & 4 & 5 & 0 & 0.1 & 0.2 & 0 & 0.511 .52\end{array}$
$\begin{array}{llll}0 & 0.2 & 0.4 & 0.6\end{array}$

(ind $/ \mathrm{m}^{3}$ )
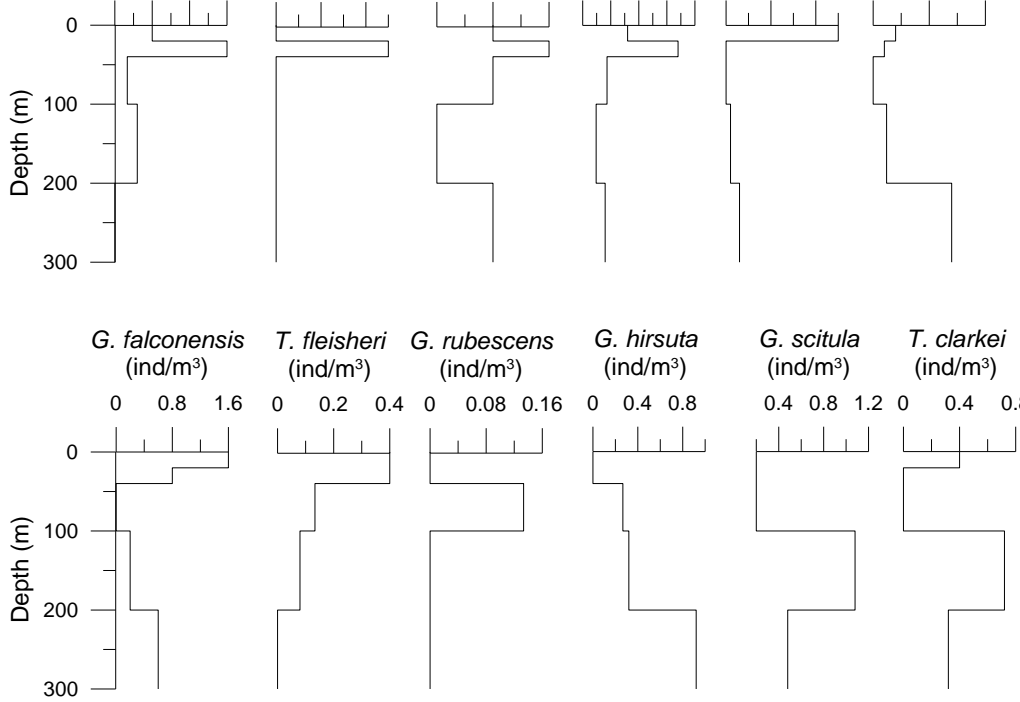

$\begin{array}{llllll}0.4 & 0.8 & 1.2 & 0 & 0.4 & 0.8\end{array}$

Figure S1. Abundance data for the most abundant species in each station. Only new data from the Azores Current/Front and Iberian Margin are presented here. 


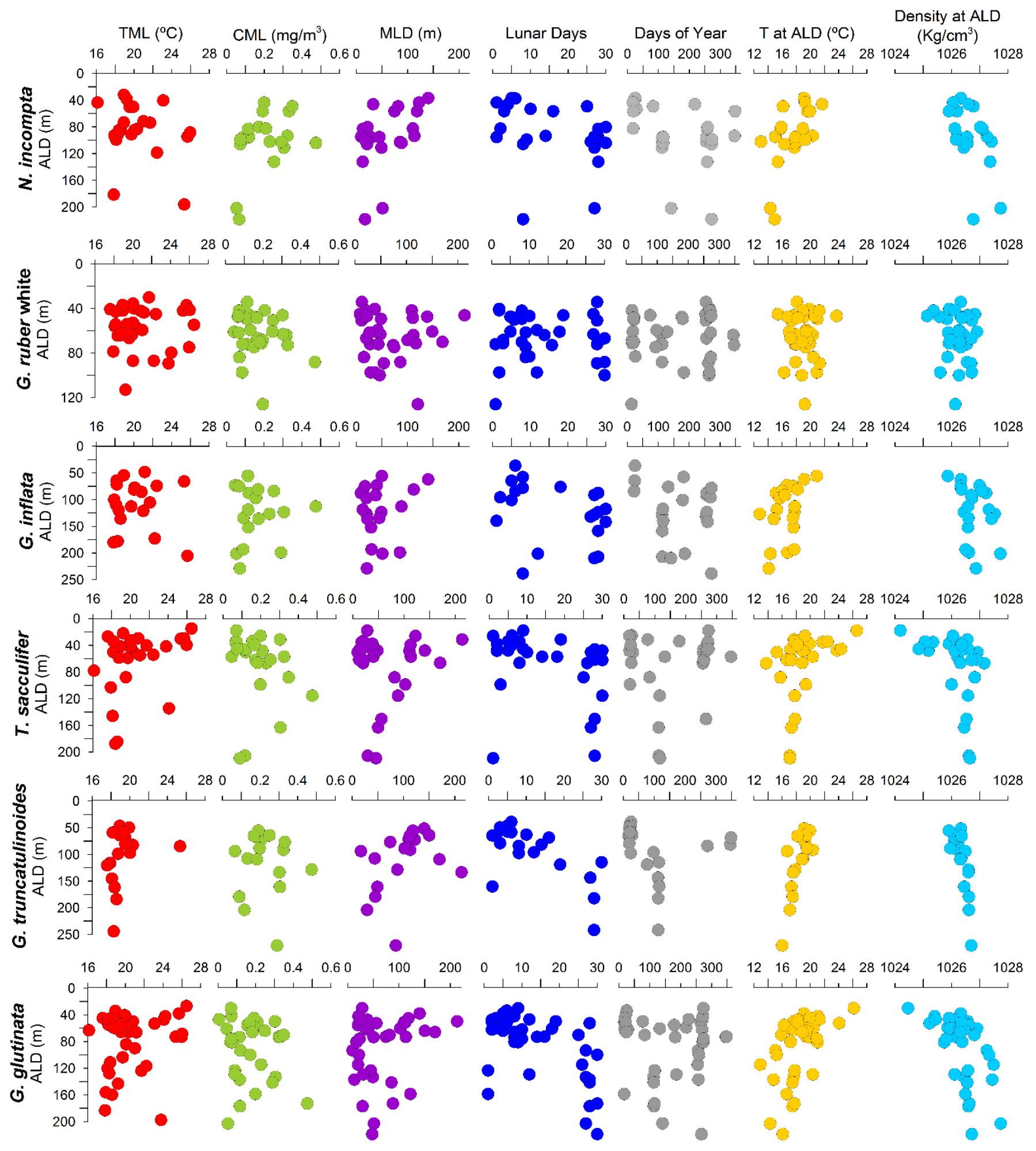




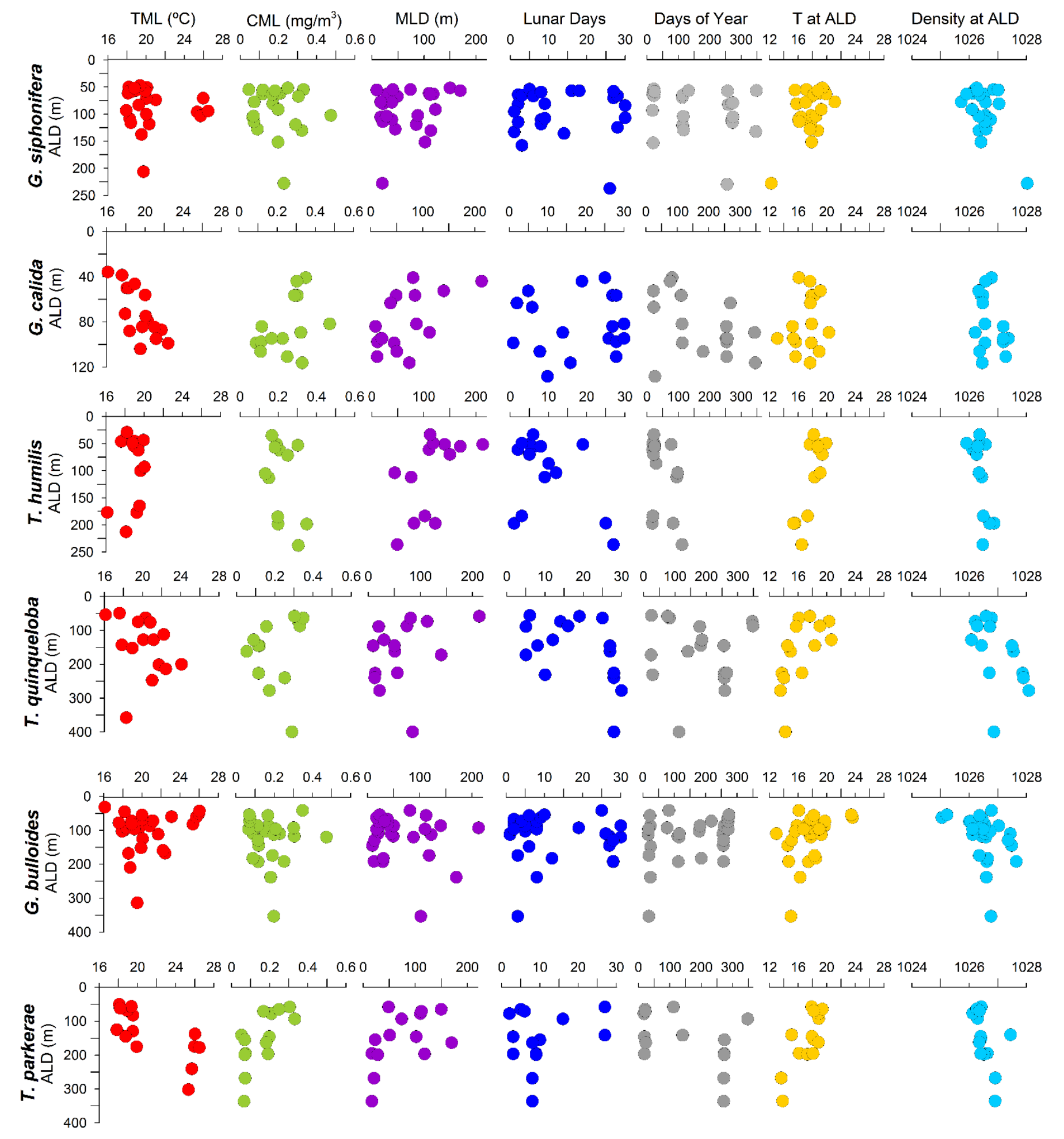




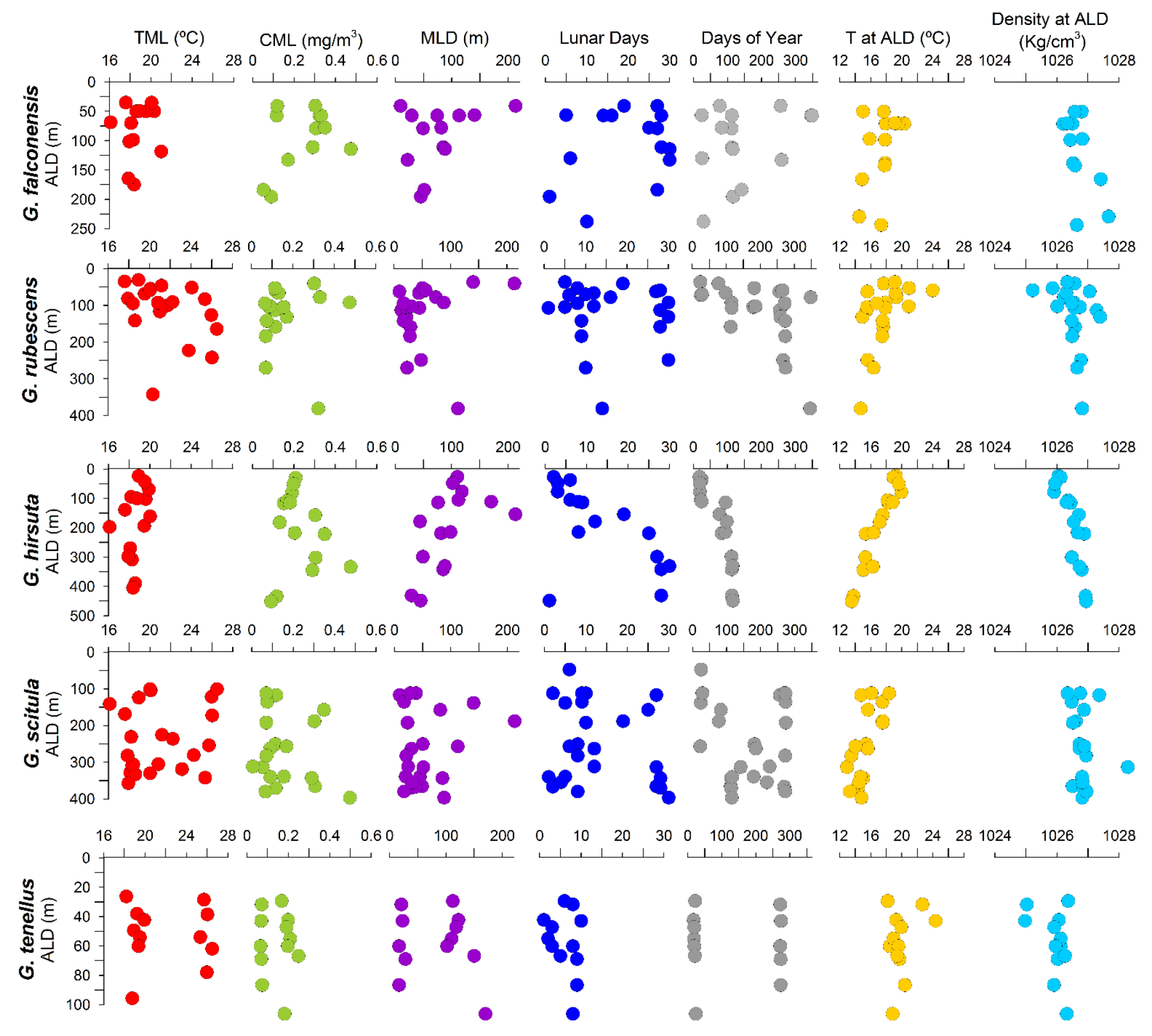

Figure S2. Observed ALD for the species with a variable habitat depth with the environmental parameters of the mix layer (temperature, chlorophyll $a$ and mixed layer depth), lunar days, days of the year, temperature at ALD and seawater density at ALD. 